\title{
Female chronic posterior urethritis is underestimated in patients with lower urinary tract symptoms
}

\author{
Juanjuan Xie ${ }^{1,2,3,4 \#}$, Xin $\mathrm{Li}^{2 \#}$, Yufang L $\ddot{u ̈}^{1,3,4}$, Caisheng Huang ${ }^{1,3,4}$, Xinyang Long ${ }^{3,4,5}$, Yong Liu ${ }^{2}$, \\ Huaxiang $\mathrm{Lu}^{6}$, Jianhua Long ${ }^{2}$, Bo Chen ${ }^{7}$, Zhigang Luo ${ }^{2}$, Zengnan Mo ${ }^{1,3,4,5,8}$ \\ ${ }^{1}$ Department of Urology, The First Affiliated Hospital of Guangxi Medical University, Guangxi Medical University, Nanning, China; ${ }^{2}$ Department \\ of Urology, The Second Affiliated Hospital, Hengyang Medical School, University of South China, Hengyang, China; ${ }^{3}$ Institute of Urology and \\ Nephrology, The First Affiliated Hospital of Guangxi Medical University, Guangxi Medical University, Nanning, China; ${ }^{4}$ Center for Genomic and \\ Personalized Medicine, Guangxi Medical University, Nanning, China; ${ }^{5}$ Guangxi Collaborative Innovation Center for Genomic and Personalized \\ Medicine, Guangxi Medical University, Nanning, China; ${ }^{6}$ School of Public Health, Guangxi Medical University, Nanning, China; ${ }^{7}$ Department of \\ Computer Center, The Second Affiliated Hospital, Hengyang Medical School, University of South China, Hengyang, China; ${ }^{8}$ Guangxi Collaborative \\ Innovation Center for Biomedicine, Guangxi Medical University, Nanning, China \\ Contributions: (I) Conception and design: J Xie, Z Mo, Z Luo; (II) Administrative support: J Xie, Z Mo, Z Luo; (III) Provision of study materials or \\ patients: J Xie, J Long; (IV) Collection and assembly of data: J Xie, X Li, B Chen, Y Liu; (V) Data analysis and interpretation: J Xie, X Long, X Lu, C \\ Huang; (VI) Manuscript writing: All authors; (VII) Final approval of manuscript: All authors. \\ \#These authors contributed equally to this work. \\ Correspondence to: Zhigang Luo. Department of Urology, The Second Affiliated Hospital, Hengyang Medical School, University of South China, \\ Hengyang 421001, China. Email: 706885399@qq.com; Zengnan Mo. Department of Urology, The First Affiliated Hospital of Guangxi Medical \\ University, Guangxi Medical University, Guangxi 530021, China. Email: mozengnan@gxmu.edu.cn.
}

Background: As one of the causes of urethral symptoms, female chronic posterior urethritis is a common and distressing disease; however, it is often neglected and misdiagnosed as overactive bladder (OAB) or interstitial cystitis/bladder pain syndrome (IC/BPS). Currently, little is known about the urothelium and lamina propria of the bladder neck and proximal urethra. Thus, identifying urethral lesions is necessary for the diagnosis and treatment of female chronic posterior urethritis. Transurethral electroresection is an effective and safe approach for treating female chronic posterior urethritis. This study sought to determine if urethral lesions are necessary for the diagnosis and treatment of female chronic posterior urethritis, and evaluate the efficacy and safety of the transurethral electroresection of mucosa and submucosa in treating female chronic posterior urethritis.

Methods: A single-center, retrospective, observational study was conducted at a teaching and referral hospital. A total of 147 female patients who had been diagnosed with chronic papillary urethritis underwent transurethral electroresection between 2015 and 2018. Each patient underwent a follow-up examination. A chart review was also performed.

Results: Patients had a mean age of 54 years (range, 23-82 years), and the average follow-up period was 54.8 months (range, 6-600 months). Urinary frequency and urgency (51.7\%) were the most common clinical manifestations of chronic posterior urethritis. Forty-two-point two percent of patients had positive urine culture results, most commonly with Mycoplasma genitalium. The cystoscopic findings revealed that chronic posterior urethritis has tuft-like, pseudopodia-like, finger-like, and follicular-like polyps and villi, and a pebble-like appearance with mucosal hyperemia. The success rate of the transurethral electroresection was $88.6 \%$, and patients showed no apparent or serious complications.

Conclusions: This study showed that female chronic posterior urethritis is a cause that contributes to LUT symptoms. Its characteristic cystoscopic appearance and biopsy play a vital role in its diagnosis. The transurethral electroresection of urethral lesions is simple, effective, and minimally invasive without any apparent complications. 
Keywords: Chronic polypoid urethritis; lower urinary tract symptoms (LUT symptoms); female posterior urethritis; transurethral electroresection; urethral syndrome

Submitted Jun 07, 2021. Accepted for publication Jul 27, 2021.

doi: $10.21037 /$ tau-21-550

View this article at: https://dx.doi.org/10.21037/tau-21-550

\section{Introduction}

Chronic posterior urethritis, which is a chronic non-specific inflammation condition of the intraurethral, results from a response to injury in the urethral mucosa (1). It has chronic posterior urethritis symptoms, including urethral pain, gross/microscopic hematuria, urinary frequency, urinary urgency, and dysuria and other lower urinary tract (LUT) symptoms (2-4). As these symptoms overlap with those of recurrent urinary tract infection, overactive bladder (OAB), and interstitial cystitis/bladder pain syndrome (IC/BPS) (5-7), chronic posterior urethritis can be misdiagnosed. Research has shown that the mucosal layer of the LUT is involved in mechanosensory functions that regulate both bladder contractile activity and the urethral sensation $(8,9)$. Changes to the mucosa are usually associated with disorders, including IC/BPS and OAB (10). However, compared to the urinary bladder, much less is known about the urothelium and lamina propria of the bladder neck and proximal urethra.

Urologists have treated female chronic urethritis from the bladder neck to the distal urethra with oral antibiotics or operations, such as urethral dilatations, internal urethrotomy, and urethral-hymenal fusion surgery (11-13). However, studies on the diagnosis and treatment of female chronic urethritis are scarce. This study examined the clinical data on transurethral electroresections that were performed on female patients suffering from chronic posterior urethritis, and who had experienced antibiotic resistance or therapy resistance and had been misdiagnosed with $\mathrm{OAB}$ or IC/BPS. We aimed to draw the attention of urologists to the relationship between urethral lesions and LUT symptoms, and to demonstrate the efficacy and safety of transurethral electroresection in treating chronic urethral lesions. We present the following article in accordance with the STROBE reporting checklist (available at https://dx.doi. org/10.21037/tau-21-550).

\section{Methods}

\section{Study population}

A single-center, retrospective, observational study was performed at a teaching and referral hospital from January 2015 to December 2018. A total of 147 female patients, who had been diagnosed with chronic papillary urethritis and undergone transurethral electroresection, were included in the study. All patients were treated with antibiotics or other methods at other hospitals, and had developed treatmentresistant urethritis.

To be eligible to participate in the study, patients had to meet the following inclusion criteria: (I) have symptoms such as urinary frequency, urinary urgency, dysuria, urethral pain, or gross/microscopic hematuria; (II) be older than 18 years and have a disease duration of no less than 6 months; and (III) have been diagnosed with chronic papillary urethritis or chronic cystitis and have undergone cystoscopy. Patients were excluded from the study if they met any of the following exclusion criteria: (I) had urinary tract cancer or traumatic urethral stricture; (II) were pregnant or giving childbirth; (III) had urethral diverticulum, refractory radio or chemotherapy-induced cystitis, and $\mathrm{OAB}$ or IC/BPS.

The clinical data were collected after this study was approved by the ethics committee of the Second Hospital of University of South China (No. 2020001). All data were collected by two people independently. All patients agreed to participate in this study and signed an informed consent form. All procedures performed in this study involving human participants were in accordance with the Declaration of Helsinki (as revised in 2013).

\section{Preoperative evaluation}

All patients underwent a standard preoperative assessment, 
Table 1 Characteristics of all patients

\begin{tabular}{|c|c|c|c|c|c|}
\hline Data item & Baseline data & $\begin{array}{l}\text { First } \\
\text { follow-up }\end{array}$ & $\begin{array}{l}\text { Final } \\
\text { follow-up }\end{array}$ & $\begin{array}{l}\text { Baseline vs. first follow-up } \\
\text { improvement rate }\end{array}$ & $\begin{array}{l}\text { Baseline vs. final follow-up } \\
\text { improvement rate }{ }^{2}\end{array}$ \\
\hline Age & $54.7 \pm 11.6$ & & & & \\
\hline $\begin{array}{l}\text { Preoperative average diseases } \\
\text { duration (months) }\end{array}$ & $54.8(6-600)$ & & & & \\
\hline Average follow-up period (months) & $29.8 \pm 8.8$ & & & & \\
\hline Average urinary frequency (day) & $11.4 \pm 2.9$ & $6.9 \pm 2.3$ & $6.8 \pm 2.3$ & $64 \%$ & $72.8 \%$ \\
\hline $\begin{array}{l}\text { Daily average number of nocturia } \\
\text { events (night) }\end{array}$ & $3.4 \pm 1.7$ & $1.3 \pm 2.2$ & $1.0 \pm 1.3$ & $60.3 \%$ & $62 \%$ \\
\hline Quality of life (QoL) score (1-7) & $5.8 \pm 0.7$ & $3.9 \pm 1.7$ & $3.5 \pm 1.7$ & $54.4 \%$ & $51.2 \%$ \\
\hline
\end{tabular}

\footnotetext{
${ }^{1,2}$, no improvement was defined as patients with symptom improvement of less than $50 \%$. Conversely, symptom improvement of more than $50 \%$ (effective improvement) and $80-100 \%$ (significant improvement) represented overall improvement, and all patients were included in the statistical analysis.
}

including a medical history, physical examination, laboratory tests, a routine urine test, a urine culture, a cystoscopy. Patients were also asked to keep a urination diary (for 72 hours), and complete a questionnaire survey, and quality of life (QoL) survey.

\section{Postoperative follow-up period}

Data were collected via a telephone call on which a questionnaire was administered, and during outpatient visits at the 6-month and final (at least 24-month) follow-up appointments after transurethral electroresection. At the visit, a symptom assessment, physical examination, and cystoscopy were performed, and a urinary diary was kept. Patients' symptom scores, $\mathrm{OAB}$ activity, and QoL questionnaire results were used to assess symptoms, including urinary urgency, daily frequency of urination, daily number of nocturia, and QoL score. Any complications related to the procedure were also assessed.

\section{Statistical analysis}

The statistical analysis was performed using SPSS ${ }^{\circledR}$ (version 26) software. The continuous variables are presented as mean \pm standard deviation $(\mathrm{SD})$. The categorical variables are presented as median (range), and number (percentage). Handling missing values, we deleted the researchers directly. A paired $t$ test was used to comparatively analyze the continuous variables. The interaction of time with treatment was examined using a 2 -way repeated analysis of variance (ANOVA) tests. If Mauchly's test of sphericity indicated a violation of the sphericity assumption for the repeated measure ANOVA tests, Greenhouse-Geisser sphericity correction was applied. A $\mathrm{P}<0.05$ was considered statistically significant.

\section{Results}

A total of 147 female patients underwent surgery. Among these 147 patients, 11 were lost during the follow-up period. The remaining 136 patients had an average age of 54 years (range, 23-82 years). The mean follow-up period was 54.8 months (range, 6-600 months). Thirtyone patients received transurethral electroresection of the papillary urethritis only, and 105 underwent transurethral electroresection of both urethral and bladder lesions. The mean duration of patients' symptoms was 20 months. The results in relation to the short-term efficacy (baseline $v s$. improvement 6-month post-surgery) and long-term efficacy (baseline $v s$. improvement at least 24-month post-surgery) of the approach are presented in Table 1.

Among the 147 patients, the most common symptoms were urinary frequency and urgency [76 (51.7\%); see Table 2]. Among the 146 patients with urine cultures, the results of $61(42.2 \%)$ patients were positive for bacterial growth. Surprisingly, the most pathogenic microorganism was Mycoplasma genitalium [37 (25.2\%)] and not Escherichia coli $[13(8.8 \%)]$. Cystoscopic examinations were performed to detect urethral lesions in all patients. Among the 147 cases, 29 (19.7\%), 85 (57.8\%), 14 (9.5\%), 
Table 2 Clinical characteristics and cystoscopic findings

\begin{tabular}{|c|c|c|c|c|c|}
\hline Clinical manifestation & Frequency & Urine culture & Frequency & Cystoscopic findings & Frequency \\
\hline $\begin{array}{l}\text { Pricking and burning pain } \\
\text { during urination }\end{array}$ & $15 / 147(10.2 \%)$ & $\begin{array}{l}\text { Streptococcus } \\
\text { agalactiae }\end{array}$ & $3 / 146(2.1 \%)$ & Pseudopodia-like polyps & $14 / 147(9.5 \%)$ \\
\hline Lower abdominal pain & $34 / 147(23.1 \%)$ & Proteus mirabilis & $1 / 146(0.7 \%)$ & Follicular-like polyps & $4 / 147(2.7 \%)$ \\
\hline \multirow[t]{3}{*}{ Pelvic pain } & $2 / 147(1.3 \%)$ & Enterococcus faecalis & $1 / 146(0.7 \%)$ & & \\
\hline & & Candida parapsilosis & $1 / 146(0.7 \%)$ & & \\
\hline & & Albicans candida & $1 / 146(0.7 \%)$ & & \\
\hline
\end{tabular}

$15(10.2 \%)$, and $4(2.7 \%)$ were found to have finger-like polyps, tuft-like polyps and villi, pseudopodia-like polyps, a pebble-like appearance with mucosal hyperemia and edema, and follicular-like polyps, respectively. One hundred and five of the 147 patients suffered from bladder lesions at the trigone and bladder neck in addition to urethral lesions (see Figure 1).

2 operations were performed; that is, the transurethral electroresection of the urethra only, or the transurethral electroresection of the urethra and bladder. The efficacy of each operation in treating different LUT symptoms was calculated. Patients in the urethral surgery group had a mean age of 54.4 years. Statistically, there were significant differences in the number of urination events, urgency, nocturia events, and QoL scores before and after surgery (i.e., at the end of the last follow-up appointment postsurgery; $\mathrm{P}<0.001$; see Figure 2 and Table 3). Patients in the urethra and bladder surgery group had a mean age of 54.0 years. Significant differences in the number of urination events, urgency, nocturia events, and QoL scores were found before and after surgery (i.e., at the end of the first or last follow-up appointment post-surgery; $\mathrm{P}<0.001$; see Figure 2 and Table 3).

As of the last follow-up date, 15 patients $(11 \%)$ had experienced complications, including urinary incontinence $(5.1 \%)$, urinary tract infection $(2.2 \%)$, urinary stricture $(1.5 \%)$, hematuria $(1.5 \%)$, and dysuria $(0.7 \%)$. Most of the adverse events were of short duration. 3 patients with stress urinary incontinence were treated by tension free vaginal tape (TVT), 4 patients with urgency urinary incontinence were symptom-free after inflammation was controlled.

\section{Discussion}

Chronic posterior urethritis is more difficult to diagnose in women than men, as its clinical symptoms are similar to those of $\mathrm{OAB}$, primary cystitis, vaginitis, and cervicitis (14) involving the LUT. The urothelium in the region of the bladder neck and proximal urethra play a role in continence and sensation $(9,15,16)$. The cause and pathogenic factors of female chronic posterior lesions are not yet completely known. It has been suggested that many factors, including non-specific infection, urethral obstruction, urethral-hymenal fusion, senile atrophy, traumatic causes, inflamm-ageing, and estrogen deficiency, may be implicated in its pathogenesis $(4,12,17,18)$. Urethritis is classified as gonococcal or nongonococcal urethritis (NGU) based on its causative organisms that may be sexually and non-sexually transmitted. The major causes of NGU are Chlamydia trachomatis and Mycoplasma genitalium, which account for $75 \%$ of cases (19). However, other pathogenic organisms include Trichomonas vaginalis, anaerobes, herpes simplex virus, and adenovirus (20). In the present study, we identified Mycoplasma as the most common etiological agent. 

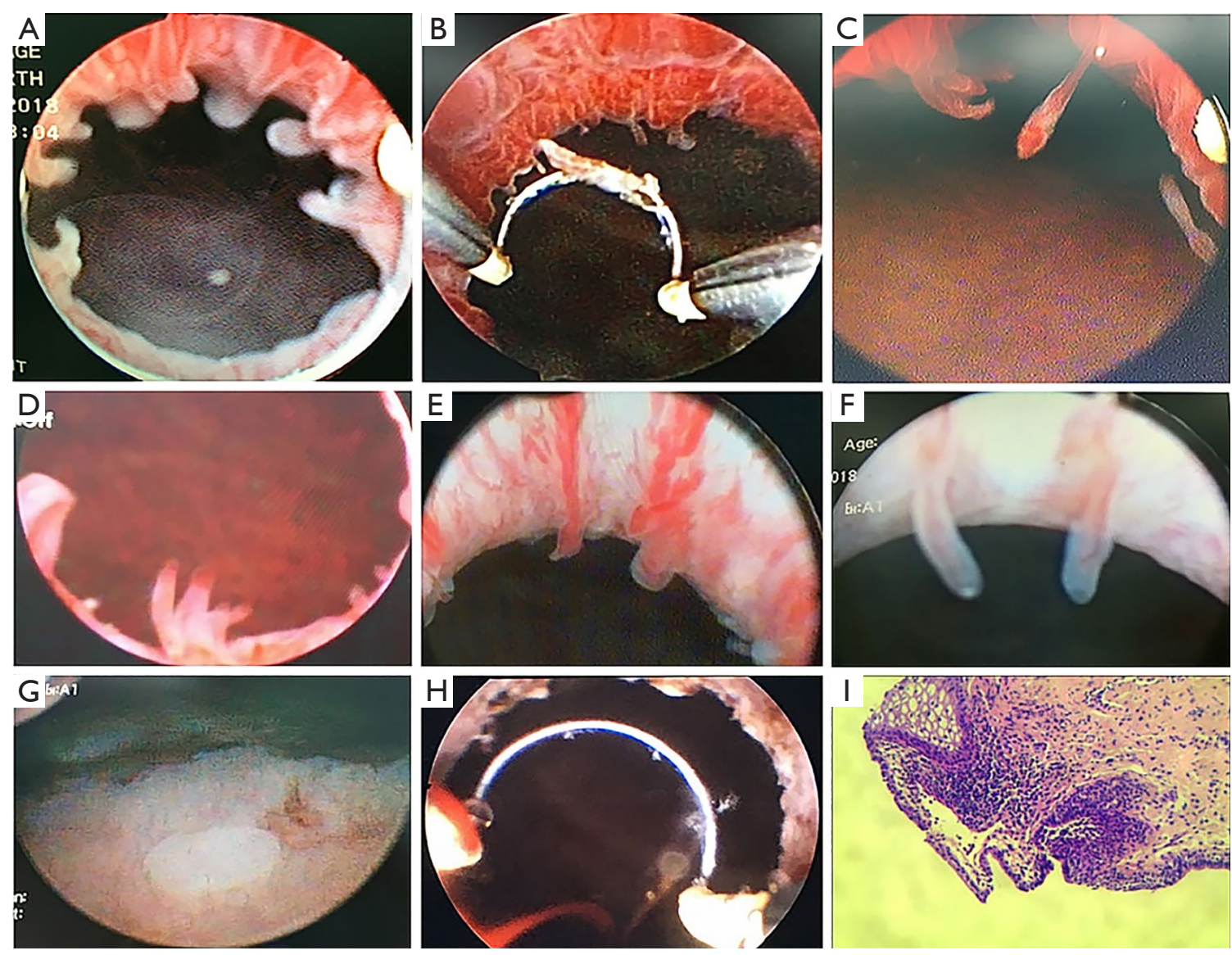

Figure 1 Cystoscopic appearance and biopsy. (A) Finger-like polyps. The locations were the posterior urethra and bladder neck. (B) Pebblelike appearance with mucosal hyperemia and edema. (C) Pseudopodia-like polyps. (D) Tuft-like polyps. (E) Follicular-like polyps. (F) Villous polyps. (G) Leukoplakia of bladder mucosa. Cystoscopic appearance of trigonitis. $(\mathrm{H})$ Cystoscopic appearance after transurethral resection. (I) Hematoxylin and eosin-stained histopathology images of urethritis (10x by light-microscope).

As these posterior urethral lesions may be caused by infection or other injury, it is necessary for women with LUT symptoms to undergo a cystoscopy to rule out urethral and bladder inflammatory lesions. The endoscopic evaluation of the LUT provides more important information than an ultrasound or urethrocystography (21). In this study, women with chronic urethritis were leukocytenegative, and exhibited only microscopic hematuria or an increase in the number of squamous cells in routine urine tests. Most urine cultures were negative for bacterial infections; however, urethral inflammatory changes were evident. The cystoscopies revealed that the center of lesions was located at the proximal urethra and bladder neck, which displayed hyperemia, edema, paler and granular changes, and polyp and villus formation(3). Posterior urethritis usually tended to be complicated by a bladder lesion called trigonitis $(22,23)$. Similar to keratinizing squamous metaplasia in the bladder, some researchers have conjectured that keratinizing squamous metaplasia is a normal variant in some conditions (24). We intend to conduct further research to determine whether such lesions exist in normal women, especially pre-menopausal women.

To identify whether lesions are the cause of female chronic posterior urethritis, we intervened by removing lesions by transurethral electroresection. Polyps and villi are the sites at which organisms persist and evade the immune response. Studies have suggested that uropathogenic bacteria can invade urothelial cells to form an intracellular bacterial community/quiescent intracellular reservoir in murine models, thus releasing strong proinflammatory factors that prompt an immune response and physiological changes in urothelial cells $(25,26)$. 

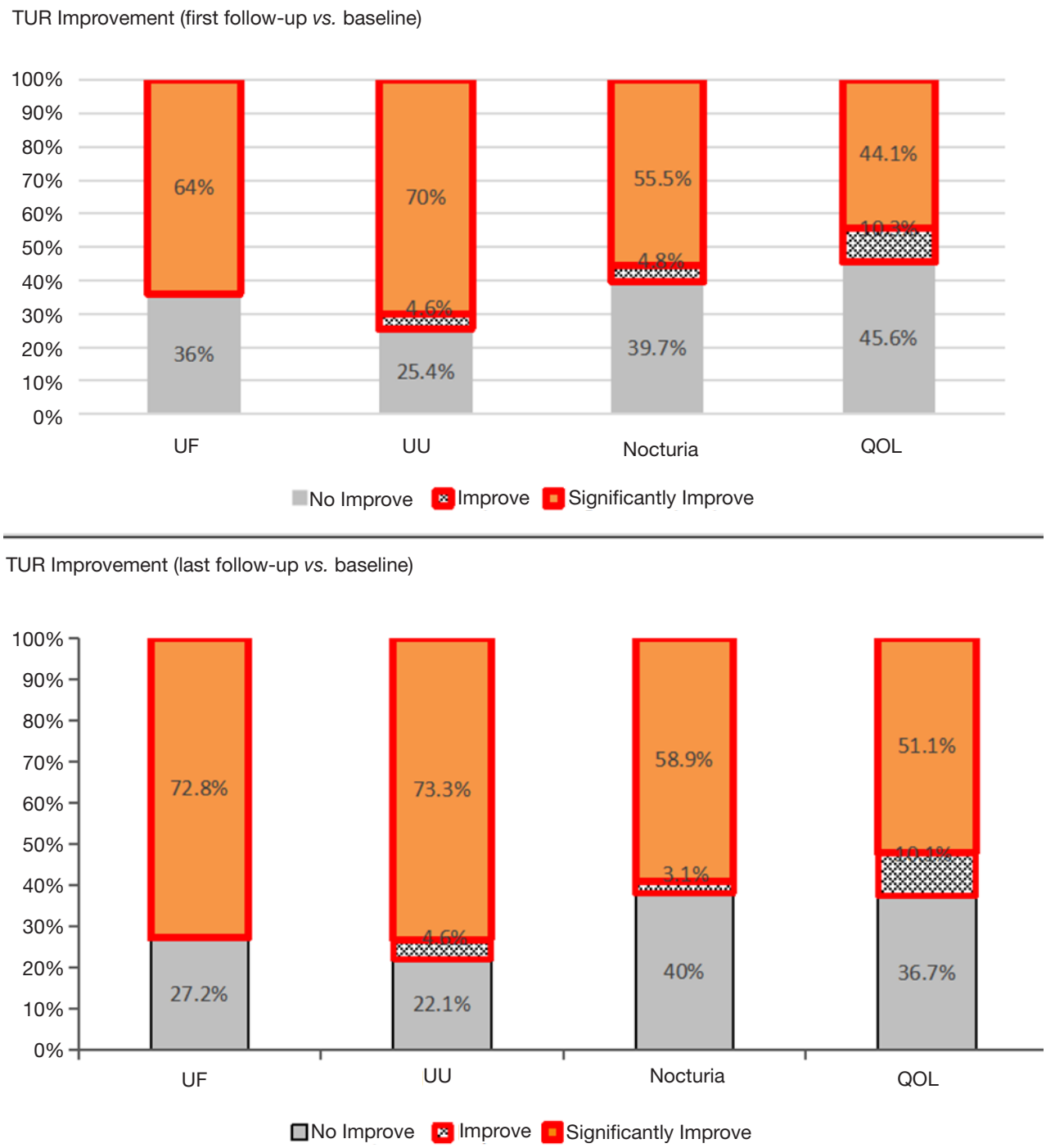

Figure 2 Efficacy evaluation of transurethral resection-entire population.

Transurethral electroresection, an effective and minimally invasive procedure with a low complication rate, can address the polyps and villus which are the sites where organisms to persist and evade the immune response. In the present study, we used the following surgical indications: (I) LUT symptoms that lasted at least 6 months; (II) urethral inflammatory changes (as observed by cystoscopy and biopsy); and (III) ineffective repeated courses of antibiotics and ineffective preventive strategies. Unlike electrofulguration (27), transurethral electroresection can perfectly resolve the mucosa and submucosa lesions of polyps and villi. In this study, we included 136 women who underwent transurethral electroresections at a single center.
The success rate of the transurethral electroresections was $88.6 \%$. The reasons for the failure of transurethral electroresection, maybe uncleaned etiology, combined other diseases such as autoimmune diseases, depression, central nervous system disorders. In order to improve the success rate and prevent the recurrence of polyps, if possible, etiology-based and combined with other treatments should be administered at the same time. After a post-transurethral electroresection follow-up period of 24 months, all preoperative lesions were resolved, and no new lesions were found. We focused on the 2 major late complications; that is, urinary incontinence $(5.1 \%)$ and urethral stricture $(1.5 \%)$. Notably, urethral stricture failed to develop to the 
Table 3 Analysis of transurethral resection of 2 groups' statistical data

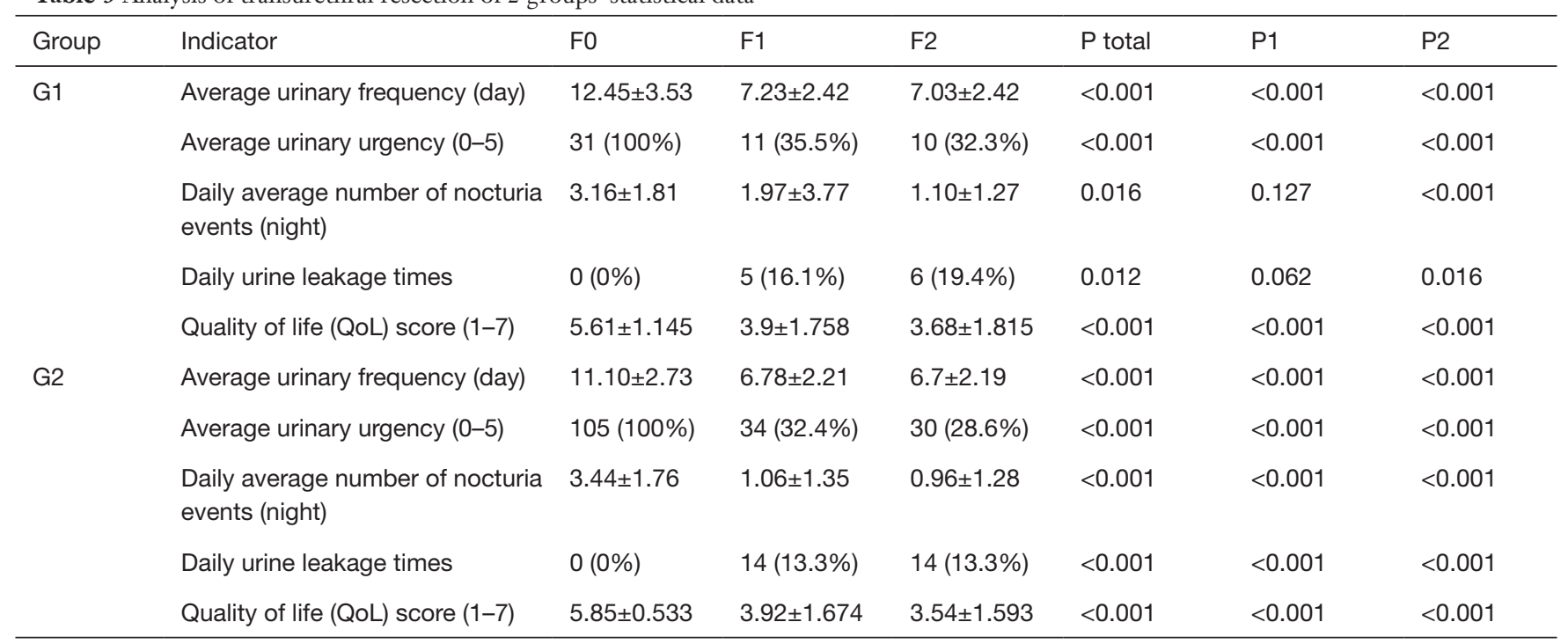

G1, transurethral resection of urethral lesion subgroup; G2, transurethral resection of urethral and bladder lesion subgroup; F0, baseline data; F1, first follow-up data; F2, last follow-up data; $P$ total, overall differences between the 3 time points; P1 (F0 vs. F1), comparison of first follow-up and baseline data; P2 (F0 vs. F2), comparison of last follow-up and baseline data.

bladder neck contracture; rather, it was safely and effectively resolved by urethral dilatation. In the present study, transurethral electroresection did not increase the incidence of stress urinary incontinence unless it occurred before the operation.

This retrospective study had several limitations, including a potential bias, reporting errors, and the need for a longer follow-up period and high-quality trials. Based on the results of this retrospective study, while transurethral electroresection can ameliorate LUT symptoms effectively, we need to further verify whether a sham treatment (e.g., a cystoscopy without resection) would lead to symptom relief by conducting a random control trial in the future.

\section{Conclusions}

This study showed that urologists need to pay attention to the diagnosis and treatment of female chronic posterior urethritis, as it has LUT symptoms. Urethral lesions found in cystoscopy may be important in diagnosing the refractory disease of LUT. Further, transurethral electroresection is a simple, effective, minimally invasive approach for treating chronic posterior urethritis that does not give rise to any apparent complications.

\section{Acknowledgments}

We would like to thank Dr. Jianjun Li (Department of Urology, The Second Affiliated Hospital, Hengyang Medical School, University of South China, Hengyang 421001, China) for his help with the data collection.

Funding: The study was supported by the Funding Project of Hengyang Science and Technology Bureau (2019jh010983).

\section{Footnote}

Reporting Checklist: The authors have completed the STROBE reporting checklist. Available at https://dx.doi. org/10.21037/tau-21-550

Data Sharing Statement: Available at https://dx.doi. org/10.21037/tau-21-550

Conflicts of Interest: All authors have completed the ICMJE uniform disclosure form (available at https://dx.doi. org/10.21037/tau-21-550). The authors have no conflicts of interest to declare.

Ethical Statement: The authors are accountable for all 
aspects of the work in ensuring that questions related to the accuracy or integrity of any part of the work are appropriately investigated and resolved. The clinical data were collected after this study was approved by the ethics committee of the Second Hospital of University of South China (No. 2020001). All data were collected by two people independently. All patients agreed to participate in this study and signed an informed consent form. All procedures performed in this study involving human participants were in accordance with the Declaration of Helsinki (as revised in 2013).

Open Access Statement: This is an Open Access article distributed in accordance with the Creative Commons Attribution-NonCommercial-NoDerivs 4.0 International License (CC BY-NC-ND 4.0), which permits the noncommercial replication and distribution of the article with the strict proviso that no changes or edits are made and the original work is properly cited (including links to both the formal publication through the relevant DOI and the license). See: https://creativecommons.org/licenses/by-nc-nd/4.0/.

\section{References}

1. Lane Z, Epstein JI. Polypoid/papillary cystitis: a series of 41 cases misdiagnosed as papillary urothelial neoplasia. Am J Surg Pathol 2008;32:758-64.

2. Baka S, Kouskouni E, Antonopoulou S, et al. Prevalence of Ureaplasma urealyticum and Mycoplasma hominis in women with chronic urinary symptoms. Urology 2009;74:62-6.

3. HODGES CV. Chronic urethritis in girls. J Am Med Assoc 1952;149:753-6.

4. Bodner DR. The urethral syndrome. Urol Clin North Am 1988;15:699-704.

5. Eapen RS, Radomski SB. Review of the epidemiology of overactive bladder. Res Rep Urol 2016;8:71-6.

6. Patnaik SS, Laganà AS, Vitale SG, et al. Etiology, pathophysiology and biomarkers of interstitial cystitis/ painful bladder syndrome. Arch Gynecol Obstet 2017;295:1341-59.

7. Ahmed H, Davies F, Francis N, et al. Long-term antibiotics for prevention of recurrent urinary tract infection in older adults: systematic review and metaanalysis of randomised trials. BMJ Open 2017;7:e015233.

8. Gosling JA, Thompson SA. A neurohistochemical and histological study of peripheral autonomic neurons of the human bladder neck and prostate. Urol Int
1977;32:269-76.

9. Birder LA, Andersson KE, Kanai AJ, et al. Urothelial mucosal signaling and the overactive bladder-ICI-RS 2013. Neurourol Urodyn 2014;33:597-601.

10. Kaur H, Arunkalaivanan AS. Urethral pain syndrome and its management. Obstet Gynecol Surv 2007;62:348-51; quiz 353-4.

11. Hedlund PO. Experience of the Rieser operation for chronic female urethritis. A follow-up study of 42 cases. Scand J Urol Nephrol 1979;13:217-9.

12. Reed JF Jr. Urethral-hymenal fusion: a cause of chronic adult female cystitis. J Urol 1970;103:441-6.

13. McLean P, Emmett JL. Internal urethrotomy in women for recurrent infection and chronic urethritis. J Urol 1969;101:724-8.

14. Moi H, Blee K, Horner PJ. Management of nongonococcal urethritis. BMC Infect Dis 2015;15:294.

15. Birder LA. Urothelial signaling. Handb Exp Pharmacol 2011;(202):207-31.

16. Khandelwal P, Abraham SN, Apodaca G. Cell biology and physiology of the uroepithelium. Am J Physiol Renal Physiol 2009;297:F1477-501.

17. Kagan R, Kellogg-Spadt S, Parish SJ. Practical Treatment Considerations in the Management of Genitourinary Syndrome of Menopause. Drugs Aging 2019;36:897-908.

18. Ligon MM, Wang C, DeJong EN, et al. Single cell and tissue-transcriptomic analysis of murine bladders reveals age- and TNF $\alpha$-dependent but microbiota-independent tertiary lymphoid tissue formation. Mucosal Immunol 2020;13:908-18.

19. Galadari I, Galadari H. Nonspecific urethritis and reactive arthritis. Clin Dermatol 2004;22:469-75.

20. Moi H, Horner PJ. 2016 European guideline on the management of non-gonococcal urethritis published. Euro Surveill 2016. doi: 10.2807/1560-7917. ES.2016.21.22.30248.

21. Chew DJ, Buffington T, Kendall MS, et al. Urethroscopy, cystoscopy, and biopsy of the feline lower urinary tract. Vet Clin North Am Small Anim Pract 1996;26:441-62.

22. Sadeghi Z, MacLennan G, Childs SJ, et al. Is trigonitis a neglected, imprecise, misunderstood, or forgotten diagnosis? Low Urin Tract Symptoms 2019;11:182-8.

23. MAAS IP. Chronic urethro-trigonitis in women. S Afr Med J 1952;26:655-7.

24. Clouston D, Lawrentschuk N. Metaplastic conditions of the bladder. BJU Int 2013;112 Suppl 2:27-31.

25. Abraham SN, Miao Y. The nature of immune responses to urinary tract infections. Nat Rev Immunol 
2015;15:655-63.

26. Ingersoll MA, Albert ML. From infection to immunotherapy: host immune responses to bacteria at the bladder mucosa. Mucosal Immunol 2013;6:1041-53.

27. Crivelli JJ, Alhalabi F, Zimmern PE. Electrofulguration

Cite this article as: Xie J, Li X, Lü Y, Huang C, Long X, Liu Y, Lu H, Long J, Chen B, Luo Z, Mo Z. Female chronic posterior urethritis is underestimated in patients with lower urinary tract symptoms. Transl Androl Urol 2021;10(8):3456-3464. doi: $10.21037 /$ tau-21-550 in the advanced management of antibiotic-refractory recurrent urinary tract infections in women. Int J Urol 2019;26:662-8.

(English Language Editor: L. Huleatt) 